\title{
Operation of a Stark decelerator with optimum acceptance
}

\author{
Ludwig Scharfenberg, ${ }^{*}$ Henrik Haak, Gerard Meijer, and Sebastiaan Y. T. van de Meerakker \\ Fritz-Haber-Institut der Max-Planck-Gesellschaft, Faradayweg 4-6, 14195 Berlin, Germany
}

(Received 24 July 2008; published 6 February 2009)

\begin{abstract}
With a Stark decelerator, beams of neutral polar molecules can be accelerated, guided at a constant velocity, or decelerated. The effectiveness of this process is determined by the six-dimensional (6D) volume in phase space from which molecules are accepted by the Stark decelerator. Couplings between the longitudinal and transverse motion of the molecules in the decelerator can reduce this acceptance. These couplings are nearly absent when the decelerator operates such that only every third electric-field stage is used for deceleration, while extra transverse focusing is provided by the intermediate stages. For many applications, the acceptance of a Stark decelerator in this so-called $s=3$ mode significantly exceeds that of a decelerator in the conventionally used $(s=1)$ mode. This has been experimentally verified by passing a beam of $\mathrm{OH}$ radicals through a 2.6-m-long Stark decelerator. The experiments are in quantitative agreement with the results of trajectory calculations, and can qualitatively be explained with a simple model for the $6 \mathrm{D}$ acceptance. These results imply that the $6 \mathrm{D}$ acceptance of a Stark decelerator in the $s=3$ mode of operation approaches the optimum value, i.e., the value that is obtained when any couplings are neglected.
\end{abstract}

DOI: $10.1103 /$ PhysRevA.79.023410

PACS number(s): 37.10.Mn, 37.20.+j

\section{INTRODUCTION}

Since its introduction in 1999, the method of Stark deceleration has developed into an established method for taming molecular beams $[1,2]$. The Stark deceleration technique combines molecular beam technology with concepts from charged particle accelerator physics. In essence, a part of a beam of neutral molecules is selected and decelerated utilizing the force that polar molecules experience in inhomogeneous electric fields. A Stark decelerator produces bunches of state-selected molecules with a computer-controlled velocity and with narrow velocity distributions. These beams are ideally suited for a variety of experiments in which the velocity of the molecules is an important parameter. Applications include the use of slow molecular beams to enhance the interaction time in spectroscopic experiments $[3,4]$ and scattering studies as a function of the collision energy [5]. When the molecules are decelerated to a near standstill, they can be loaded and confined in traps $[6,7]$. This allows the observation of molecules in complete isolation for times up to several seconds, and enables the investigation of molecular properties in great detail [8-10].

For many of these applications it is crucial that the number density of the decelerated packets of molecules is further increased. Higher densities of decelerated molecules will improve the statistics in metrology experiments and can be decisive, for instance for the observation of (in)elastic scattering or reactive collisions in crossed molecular beam experiments. Higher densities in the trap are also a prerequisite for the future application of cooling schemes like evaporative cooling, needed to reach the regime of degenerate dipolar quantum gases [12].

The number density of decelerated molecules that can be reached at the exit of the decelerator is limited by the initial

\footnotetext{
*Author to whom correspondence should be addressed. ludwig @ fhi-berlin.mpg.de
}

phase-space density in the molecular beam and by the sixdimensional (6D) phase-space acceptance of the decelerator. The latter is defined as the volume in 6D phase space-the product of the volume in real space and in velocity spacefrom which stable trajectories through the decelerator originate. In most Stark deceleration experiments to date, molecular beams with a low initial velocity are slowed down using decelerators with a rather limited number of electric field stages. Hence, molecular beams are typically released from a cooled pulsed valve using $\mathrm{Xe}$ or $\mathrm{Kr}$ as a carrier gas. The use of $\mathrm{Xe}$ or $\mathrm{Kr}$ and the cooling of the pulsed valve strongly enhances cluster formation, however, and is generally regarded to be nonideal for a molecular beam expansion. Moreover, these decelerators are usually operated at large phase angles. The phase angle $\phi_{0}$ is defined by the position of the synchronous molecule at the moment the fields are switched [11] and determines the deceleration rate per electric field stage. It ranges from $0^{\circ}<\phi_{0}<90^{\circ}$ for deceleration, while acceleration occurs from $-90^{\circ}<\phi_{0}<0^{\circ}$. While for increasing values of $\left|\phi_{0}\right|$ the deceleration rate gets larger, the longitudinal phase-space acceptance gets smaller.

An obvious route to higher number densities of decelerated molecules is thus the use of seed gases of lower mass (preferably $\mathrm{Ne}$ or $\mathrm{Ar}$ ) in room-temperature expansions and the use of low phase angles in the decelerator. Together this implies, however, that (much) longer Stark decelerators need to be constructed to compensate for both the higher initial velocity and the lower deceleration rate. It is not a priori clear whether one can actually transport molecules through such long decelerators without significant losses. If only molecular trajectories along the molecular beam axis are considered, the length of the decelerator is inconsequential, as the deceleration process is subject to phase stability $[11,13]$. In reality, the molecules in the beam have off-axis position and velocity components, however. The transverse electric field gradients in the decelerator drive the molecules back toward the molecular beam axis. The resulting transverse oscillatory motion is strongly coupled to the longitudinal (forward) motion and can result in a reduction of the $6 \mathrm{D}$ 
phase-space acceptance of the decelerator [14]. Numerical simulations indicate that this coupled motion does not affect the overall performance of the relatively short Stark decelerators that have been used and operated at high phase angles thus far, but that it can severely affect the performance of longer decelerators that are operated at low phase angles. In the ideal case, the longitudinal and transverse motions in the Stark decelerator are completely uncoupled. This can be achieved by constructing decelerators with dedicated, spatially separated, elements for focusing and deceleration $[15,16]$, as is common practice in charged particle accelerators [17]. The required electrode geometries make the decelerator rather complex, however $[16,18]$.

In this paper, we exploit that in a Stark decelerator with the original electrode geometry, i.e., the geometry as used in Ref. [1], the coupling between the longitudinal and transverse motion can be significantly reduced when the decelerator is operated in the so-called $s=3$ mode [19]. In this mode, only every third electric field stage is used for deceleration, while extra transverse focusing is provided by the intermediate stages. We demonstrate and quantify that for many applications, the acceptance of the Stark decelerator in the $s$ $=3$ mode significantly exceeds that of a decelerator in the conventionally used $(s=1)$ mode of operation. The improved performance of the $s=3$ operation mode was demonstrated earlier for guiding at a constant velocity $\left(\phi_{0}=0^{\circ}\right)$ [14] and for deceleration in a relatively short decelerator at high phase angles [16]. However, the latter experimental arrangement did not allow for a direct comparison between the $s=1$ and 3 modes of operation, and the advantages of the $s=3$ mode of operation for a wide range of parameters cannot be inferred from these studies. Here we present experiments in which a beam of $\mathrm{OH}$ radicals passes through a 2.6-m-long Stark decelerator consisting of 316 electric field stages. In this machine, the $\mathrm{OH}$ radicals can be detected either after 103 or after 316 electric field stages, enabling a direct comparison between the $s=1$ and 3 modes of operation under otherwise identical conditions, in particular using the same phase angle $\phi_{0}$. This direct comparison allows us to draw firm conclusions on the usefulness of the $s=3$ mode of operation for a wide range of experimental parameters. The experimental results are in quantitative agreement with the results of trajectory calculations, and can be explained qualitatively with a simple model for the 6D acceptance.

\section{EXPERIMENT}

\section{A. Experimental setup}

The experimental setup is shown schematically in Fig. 1. A pulsed beam of $\mathrm{OH}$ radicals is produced via ArF-laser $(193 \mathrm{~nm})$ dissociation of $\mathrm{HNO}_{3}$ seeded in an inert gas. The dissociation is carried out inside a quartz capillary that is mounted on the orifice of a pulsed valve (General Valve, Series 99). The experiment runs at a repetition frequency of $10 \mathrm{~Hz}$. During the supersonic expansion, the majority of the $\mathrm{OH}$ radicals cool to the lowest rotational and vibrational level of the $X^{2} \Pi_{3 / 2}$ spin-orbit manifold of the electronic ground state. This population is equally distributed over the two $\Lambda$-doublet components of the $J=3 / 2$ level. Only $\mathrm{OH}$

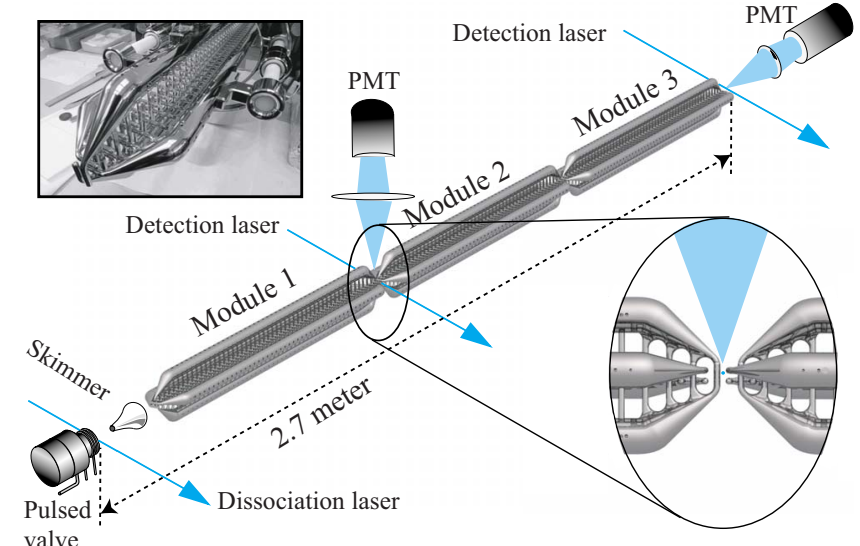

FIG. 1. (Color online) Scheme of the experimental setup. A pulsed beam of $\mathrm{OH}$ radicals is produced via photodissociation of $\mathrm{HNO}_{3}$ seeded in $\mathrm{Xe}, \mathrm{Kr}$, or Ar. The beam of $\mathrm{OH}$ radicals passes through a 2.6-m-long Stark decelerator that consists of three modules of $\sim 100$ stages each. The $\mathrm{OH}$ radicals can be state-selectively detected using a laser-induced fluorescence scheme at the end of the decelerator, and in the region between the first two modules. In the top inset, a photograph of a decelerator module is shown.

molecules in the upper $\Lambda$-doublet component are low-fieldseeking, and are of relevance to the experiments discussed here. This component splits into an $M_{J} \Omega=-3 / 4$ and an $M_{J} \Omega=-9 / 4$ component in an electric field [20]. Molecules in the $M_{J} \Omega=-9 / 4$ component experience a Stark shift that is a factor of 3 larger than the Stark shift experienced by molecules in the $M_{J} \Omega=-3 / 4$ component.

After passage through a skimmer with a 2-mm-diam opening, the molecular beam enters the differentially pumped decelerator chamber. The skimmer is mounted on a compact gate valve [21], enabling the venting of the source chamber while keeping the decelerator chamber under vacuum. The beam enters the Stark decelerator $60 \mathrm{~mm}$ from the nozzle orifice. The Stark decelerator consists of three modules that are mechanically and electrically decoupled from each other. The first two modules consist of 104 electrode pairs (i.e., 103 electric field stages) each, whereas the last module contains 109 electrode pairs. These electrode pairs consist of two parallel 4.5 -mm-diam stainless steel electrodes that are placed symmetrically around the molecular beam axis, providing a $3 \mathrm{~mm}$ gap for the molecular beam to pass through. Adjacent electrode pairs are alternately horizontally and vertically oriented [11], such that a $3 \times 3 \mathrm{~mm}^{2}$ opening area remains for the molecular beam. The electrodes of all horizontal (vertical) pairs within each module are electrically connected and switched simultaneously to high voltage. The center-to-center distance $(L)$ of electrodes of adjacent pairs is $8.25 \mathrm{~mm}$, and the three modules are carefully aligned to also maintain this distance between the electrode pairs of adjacent modules. The first and last eight electrode pairs of each module are mounted on conically shaped rods, as shown enlarged in Fig. 1. This design provides excellent optical access for fluorescence collection in between adjacent decelerator modules. It also allows the exits of two Stark decelerators in a crossed beam arrangement to be brought close together. 


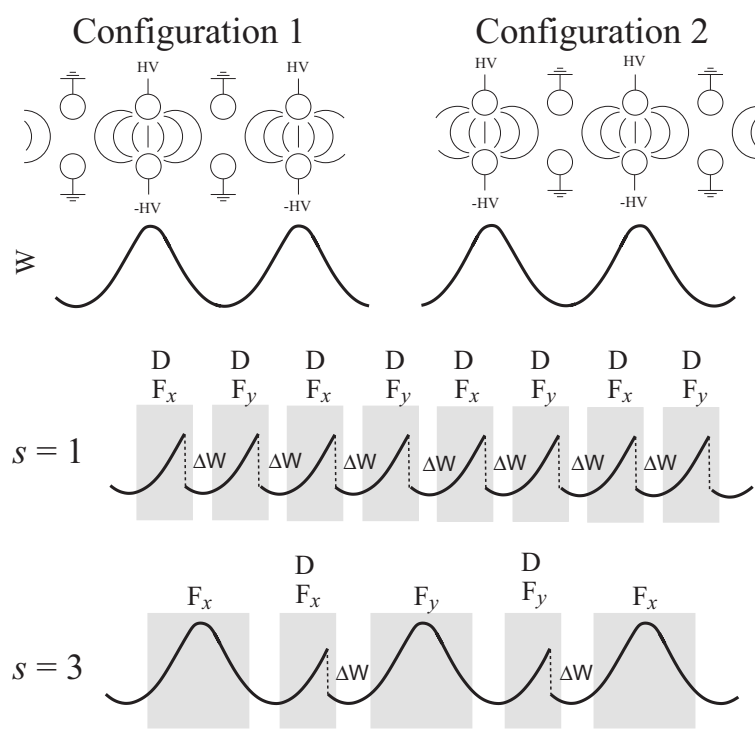

FIG. 2. Schematic representation of the two electric-field configurations that are used in the deceleration process, together with the potential energy $W$ for an $\mathrm{OH}$ molecule along the molecular beam axis. By switching between the two field configurations when the molecules are at the positions indicated by the vertical dashed lines, an amount of kinetic energy $\Delta W$ is removed from the molecules. In the conventional $(s=1)$ mode of operation, each electricfield stage is used simultaneously for deceleration (D) and focusing in alternating transverse directions $\left(F_{x}, F_{y}\right)$. In the $s=3$ mode of operation, only every third stage is used for combined deceleration and transverse focusing, while the intermediate stages provide additional focusing.

The electric field in the decelerator is switched back and forth between two different configurations, which are shown schematically in Fig. 2. In each configuration, the opposing electrodes of every other electrode pair are at $\pm 20 \mathrm{kV}$, while the remaining electrodes are grounded. Switching between the two configurations is performed using fast air-cooled high voltage switches (Behlke HTS 301-03-GSM). To minimize the power dissipation per switch, each module is connected to four separate high voltage switches. Each switch is connected to its power supply via a $0.5 \mu \mathrm{F}$ capacitor bank, limiting the voltage drop during a deceleration cycle to less than $5 \%$. For the conventional mode of operation of a Stark decelerator, the $s=1$ mode, the voltages are switched every time the molecules approach the pair of electrodes that are on high voltage [11]. In this case, the molecules are simultaneously decelerated and transversally focused in every electric field stage. When the decelerator is operated in the $s=3$ mode, the voltages are switched only after every third stage. In this case, only every third stage is used for combined deceleration and transverse focusing, while the intermediate stages provide additional focusing.

The $\mathrm{OH}$ radicals can be state-selectively detected using an off-resonant laser-induced fluorescence (LIF) detection scheme at two different positions along the beam line. The first detection zone is located between the first two modules and the second one is $18 \mathrm{~mm}$ downstream from the last module, enabling the detection of $\mathrm{OH}$ radicals after 103 or 316 electric field stages, respectively. The $282 \mathrm{~nm}$ radiation of a

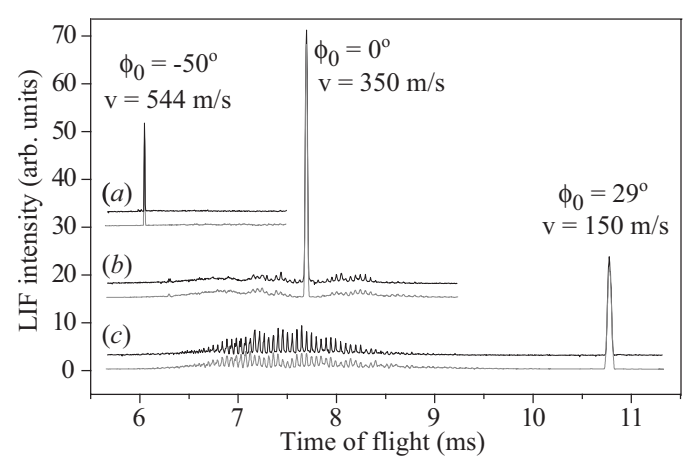

FIG. 3. Time-of-flight profiles of $\mathrm{OH}$ radicals, recorded at the exit of the 316 stage Stark decelerator. The Stark decelerator is operated at the $s=3$ mode, and accelerates [curve $(a)$ ], guides [curve $(b)$ ], or decelerates [curve $(c)$ ] a packet of $\mathrm{OH}$ radicals with an initial velocity of $350 \mathrm{~m} / \mathrm{s}$. The TOF profiles that result from simulations of the experiment are shown underneath the experimental profiles.

pulsed dye laser crosses the molecular beam in either one of the detection regions at right angles, and saturates the (spectroscopically not resolved) $Q_{21}(1)$ and $Q_{1}(1)$ transitions of the $A^{2} \Sigma^{+}, v=1 \leftarrow X^{2} \Pi_{3 / 2}, v=0$ band. The fluorescence occurs predominantly on the $A^{2} \Sigma^{+}, v=1 \rightarrow X^{2} \Pi, v=1$ transition around $313 \mathrm{~nm}$. Stray light from the laser is minimized by passing the laser beam through light baffles between the entrance and exit windows, and by optical filtering in front of the photomultiplier tube (PMT).

In the experiments, the seed gases $\mathrm{Xe}, \mathrm{Kr}$, or Ar are used. The mean initial beam velocities and velocity distributions are deduced from time-of-flight (TOF) measurements of the $\mathrm{OH}$ beam when all electrode pairs of the decelerator are simultaneously charged to $\pm 7 \mathrm{kV}$. The mean velocities for the beam are 350,430 , and $590 \mathrm{~m} / \mathrm{s}$ for $\mathrm{Xe}, \mathrm{Kr}$, and Ar, respectively, with a full width at half-maximum (FWHM) velocity spread of about 15-20\% for all seed gases.

In Fig. 3, the intensity of the LIF signal of a beam of $\mathrm{OH}$ $(J=3 / 2)$ radicals seeded in Xe is shown as a function of time after firing the dissociation laser using different deceleration sequences. The $\mathrm{OH}$ radicals are detected using the second LIF detection unit, and the Stark decelerator is operated in the $s=3$ mode. In curve $(b)$, the TOF profile is shown that is obtained when the decelerator is operated at a phase angle of $\phi_{0}=0^{\circ}$, corresponding to guiding a packet of $\mathrm{OH}$ radicals at a constant velocity. A packet of $\mathrm{OH}$ radicals with a mean velocity of $350 \mathrm{~m} / \mathrm{s}$ is selected, transported through the 2.6-m-long decelerator, and arrives in the detection region some $7.6 \mathrm{~ms}$ after its production, with a FWHM of the arrival time distribution of $25 \mu \mathrm{s}$. The measurements shown in curve $(a)$ are obtained with the decelerator operating at a phase angle of $\phi_{0}=-50^{\circ}$, accelerating a packet of $\mathrm{OH}$ radicals from an initial velocity of $350 \mathrm{~m} / \mathrm{s}$ to a final velocity of $544 \mathrm{~m} / \mathrm{s}$. There is no signature of the part of the molecular beam that is not accelerated. This is also expected as the electrodes of the decelerator are switched to ground when the accelerated packet exits the decelerator, about $1.5 \mathrm{~ms}$ before the remainder of the beam pulse would arrive in the detection region. Curve $(c)$ shows the TOF profile that is obtained when the decelerator is operated at a phase angle of $\phi_{0}$ 


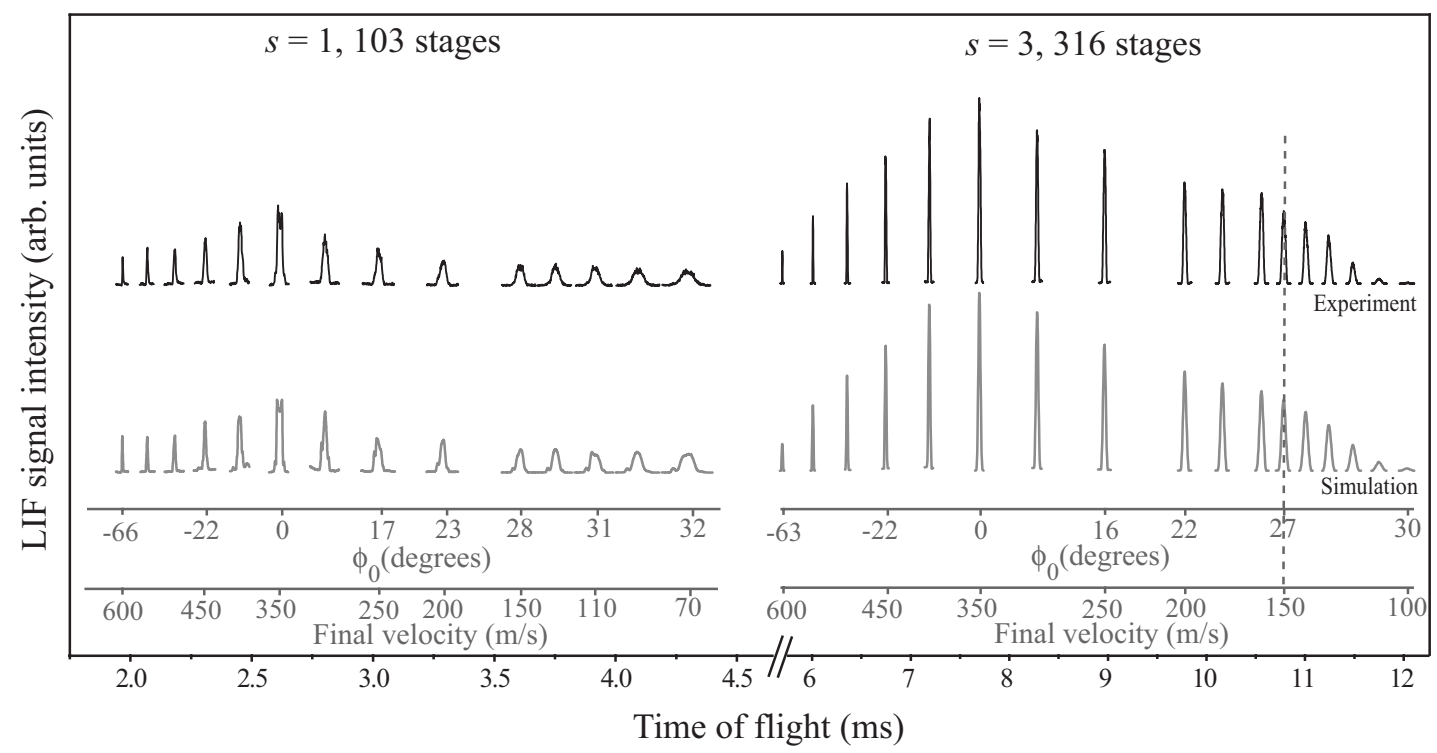

FIG. 4. Time-of-flight profiles of $\mathrm{OH}$ radicals that exit the Stark decelerator using the $s=1$ and 3 mode of operation. The OH radicals are detected after 103 and after 316 electric field stages for $s=1$ and 3, respectively. The measurements are recorded under otherwise identical conditions, and are shown on the same vertical scale. The beam of $\mathrm{OH}$ radicals has a mean initial velocity of $350 \mathrm{~m} / \mathrm{s}$. The mean final velocity of the molecules and the phase angle used are indicated for selected profiles.

$=29^{\circ}$ to decelerate a packet of $\mathrm{OH}$ radicals from 350 to $150 \mathrm{~m} / \mathrm{s}$. The decelerated molecules exit the decelerator about $10.7 \mathrm{~ms}$ after production, about $3 \mathrm{~ms}$ after the arrival of the undecelerated part of the beam.

The experimental TOF profiles are in excellent agreement with the profiles that result from three-dimensional trajectory simulations of the experiment that are shown underneath the experimental profiles. In these and in all subsequent simulations, the individual contributions of the $M_{J} \Omega=-3 / 4$ and $-9 / 4$ components to the LIF signal intensity are taken into account.

\section{B. Comparing the $s=1$ and 3 modes of operation}

In Fig. 4, two series of TOF profiles are shown that allow a direct comparison between the performance of a Stark decelerator in the $s=1$ and 3 operation mode under otherwise identical conditions. In both series, the Stark decelerator is programmed to accelerate, guide, or decelerate a packet of $\mathrm{OH}$ radicals with a mean initial velocity of $350 \mathrm{~m} / \mathrm{s}$ to a final velocity that is in the $70-600 \mathrm{~m} / \mathrm{s}$ range $\left(-65^{\circ}<\phi_{0}<32^{\circ}\right)$. Only that part of each TOF profile that contains the signature of the packet at the final velocity is shown. In the left and right panels, the series of profiles are shown that are obtained when the decelerator is operated using the $s=1$ and the $s$ $=3$ mode, detecting the $\mathrm{OH}$ radicals after 103 and 316 stages, respectively. The (almost) factor of 3 difference in the number of stages results in (almost) identical phase angles for the Stark decelerator to produce a given final velocity in both series. The phase angle that is used, and the final velocity of the packet, is indicated for selected profiles in both panels. To enable a direct comparison between the two modes of operation, both series are plotted on the same vertical scale. For this, the relative detection efficiency in the two LIF zones is experimentally determined by measuring $\mathrm{OH}$ radi- cals at both detection locations when the Stark decelerator is operated at $s=3, \phi_{0}=0^{\circ}$. The overall scaling factor that is thus determined is correct if we assume that for the $s=3$, $\phi_{0}=0^{\circ}$ mode of operation, the number density of the packet of $\mathrm{OH}$ radicals does not decline when progressing from the first to the second LIF zone, and that the relative detection efficiency is independent of the velocity of the $\mathrm{OH}$ radicals. The validity of both assumptions is checked experimentally and verified by numerical simulations.

When the decelerator is operated using $s=1$, the signal intensity for $\phi_{0}=0^{\circ}$ is about a factor of 2.5 lower than the signal intensity for $s=3, \phi_{0}=0^{\circ}$. The signal intensity for the $s=1$ appears rather constant for the different values of $\phi_{0}$ that are used. For the $s=3$ mode of operation, it is observed that the signal intensity for $\phi_{0} \neq 0^{\circ}$ gradually reduces for increasing absolute values of the phase angle, following the reduced acceptance of the decelerator. For final velocities below $150 \mathrm{~m} / \mathrm{s}$, indicated by the dashed line in Fig. 4, a sharp reduction of the signal intensity is observed even though the change in phase angle is only very small. This reduction is due to excessive transverse focusing for low velocities, and will be discussed in more detail in Sec. II D. The signal intensities for the $s=1$ and 3 modes of operation are about equal for $\phi_{0}=-65^{\circ}$. Both series of TOF profiles are in excellent agreement with profiles that result from simulations of the experiment that are shown underneath the experimental profiles. It is noted that the low field seeking $M_{J} \Omega$ $=-3 / 4$ component only contributes to the TOF profiles for $-30^{\circ} \leqslant \phi_{0} \leqslant 30^{\circ}$.

The gain of the $s=3$ mode with respect to the $s=1$ mode of operation, defined as the ratio of the maximum signal intensities of the decelerated packets at a given final velocity, is shown as a function of the final velocity in Fig. 5. For this, the data presented in Fig. 4 are used. For selected velocities, the phase angle that is used for $s=3$ operation is indicated; 


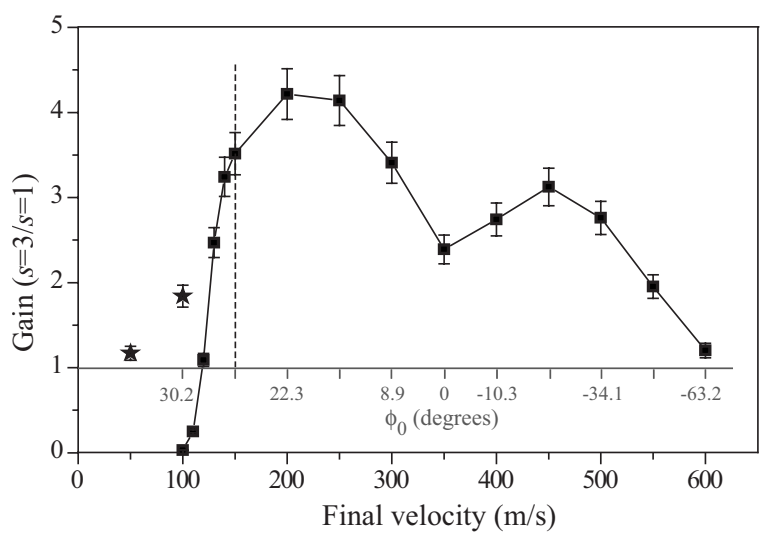

FIG. 5. The ratio of the maximum signal intensities (squares, connected by straight line segments) for $s=3$ operation versus $s$ $=1$ operation as a function of the final velocity. The two additional data points (stars) apply to a bimodal operation of the decelerator (see text for details).

the phase angle that is used for $s=1$ operation differs only slightly from this value. It is seen that for $\phi_{0}=0^{\circ}$, i.e., at a final velocity of $350 \mathrm{~m} / \mathrm{s}$, the gain is 2.4 , consistent with previous studies $[16,19]$. When the beam is accelerated to $450 \mathrm{~m} / \mathrm{s}\left(\phi_{0}=-21.7^{\circ}\right)$, a gain up to 3 is observed. For higher velocities, the gain gradually reduces and reaches 1.2 for $600 \mathrm{~m} / \mathrm{s}\left(\phi_{0}=-63.2^{\circ}\right)$. A gain up to 4.2 is observed when the beam is decelerated to $200 \mathrm{~m} / \mathrm{s}\left(\phi_{0}=22.3^{\circ}\right)$. Below $150 \mathrm{~m} / \mathrm{s}\left(\phi_{0}=27.0^{\circ}\right)$, the gain drops fast and reaches 1.0 for a final velocity of $120 \mathrm{~m} / \mathrm{s}$.

\section{The $s=1$ mode of operation at low phase angles}

In this section, we want to address the question of whether, for a fixed initial and final velocity, the number density of decelerated molecules can also be increased by using lower phase angles in the $s=1$ mode of operation. For this, we compare the deceleration with 103 stages at a certain phase angle to the deceleration with 316 stages at about onethird of this phase angle. The data for the deceleration at the $s=1$ mode with 103 deceleration stages have already been shown and discussed in the preceding section. The complimentary data for the deceleration at the $s=1$ mode with 316 deceleration stages have been measured as well; a beam of $\mathrm{OH}$ radicals with the same initial velocity of $350 \mathrm{~m} / \mathrm{s}$ is decelerated or accelerated to the same final velocities between 70 and $600 \mathrm{~m} / \mathrm{s}$. The phase angles used for these measurements are very low, ranging from $-21.7^{\circ}$ to $10.9^{\circ}$. Again, the signal intensity that is observed with guiding at the $s$ $=3$ mode is used to calibrate the measurements with 316 stages relative to the ones with 103 stages.

For the operation in the $s=1$ mode, the gain in using 316 stages compared to using 103 stages is shown as a function of the final velocity in the lower curve in Fig. 6. This gain is determined as the ratio of the signal intensities of the decelerated packets at a given final velocity, and lies between 0.5 and 0.7 throughout. Since this gain stays smaller than 1 in the considered velocity range, it is evident that for the chosen parameters the number density of decelerated molecules can-

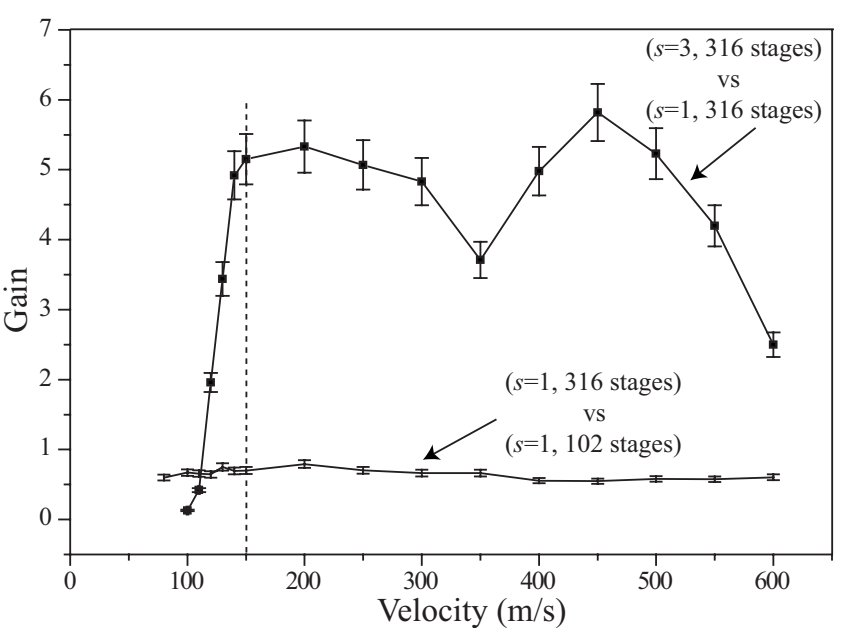

FIG. 6. Lower curve: The ratio of the signal intensity for $s=1$ operation of a decelerator with 316 vs 103 stages as a function of the final velocity. Upper curve: The ratio of the signal intensity using a 316 stage decelerator operating on the $s=3$ vs the $s=1$ mode.

not be increased by the use of lower phase angles. This perhaps counterintuitive finding is explained by the presence of inherent instabilities in the $s=1$ mode, which manifest themselves more strongly during the increased time spent in the decelerator.

We can now also address the question of whether, given a Stark decelerator of a certain length, the maximum number density of decelerated molecules is obtained using the $s=3$ mode at a certain phase angle or using the $s=1$ mode at about one-third of this phase angle. For the 316 stage decelerator, the resulting gain of the $s=3$ versus the $s=1$ mode follows directly from the curve shown in Fig. 5 and the lower curve in Fig. 6, and is shown as the upper curve in Fig. 6. Operation on the $s=3$ mode is seen to be about a factor 5 better than on the $s=1$ mode, provided the final velocity is larger than the threshold velocity.

\section{Excessive focusing at low velocities}

The rather abrupt decrease in the number density of decelerated molecules for velocities below $150 \mathrm{~m} / \mathrm{s}$ as shown in the right-hand panel of Fig. 4 can be understood qualitatively as follows. During their flight through the decelerator, molecules are alternatingly focused in each transverse direction. When the focusing force acts in one transverse direction, molecules experience to a good approximation no focusing or defocusing force in the orthogonal transverse direction (the molecules actually experience a small defocusing force in the orthogonal direction). As long as the characteristic wavelength $\lambda$ of the transverse oscillatory motion is much larger than the periodicity of the transverse focusing force, molecules will follow stable trajectories through the decelerator. The wavelength $\lambda$ is given by $\lambda=\left\langle v_{z}\right\rangle 2 \pi / \Omega_{y}$, where $\left\langle v_{z}\right\rangle$ and $\Omega_{y}$ are the mean longitudinal velocity and the mean transverse oscillation frequency of the packet of molecules, respectively. The periodicity of the focusing force is given by $2 s L$, where $L$ is the center-to-center distance of 

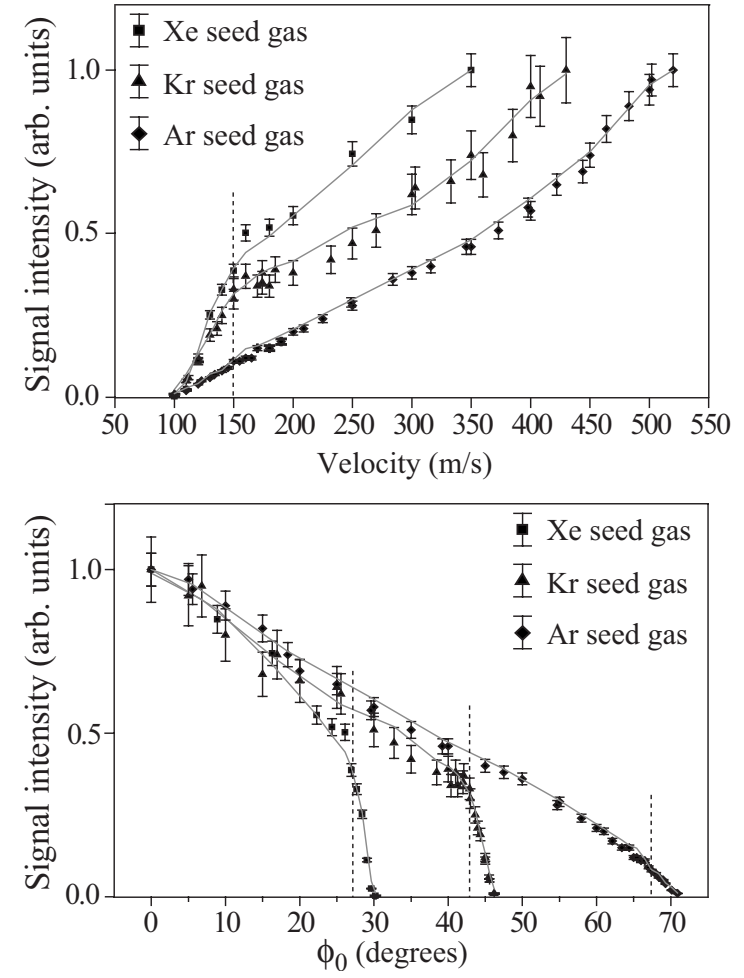

FIG. 7. Maximum signal intensity of decelerated packets of $\mathrm{OH}$ radicals as a function of the final velocity (upper panel) and as a function of $\phi_{0}$ (lower panel), using a 316 stage decelerator operating at the $s=3$ mode. Beams of $\mathrm{OH}$ radicals with three different initial velocities, produced by seeding in $\mathrm{Xe}, \mathrm{Kr}$, or Ar, have been used. The intensities that are obtained from numerical simulations of the experiment are shown as solid lines.

adjacent electrode pairs. For high velocities, therefore, stable trajectories are expected. For low velocities, however, $\lambda$ becomes ever closer to $2 s L$, and molecules will get more tightly transversely focused. The molecular trajectories will then exhibit ever larger deviations from the molecular beam axis, and the molecules will eventually crash onto the electrodes. For a given electric field distribution in a Stark decelerator, the resulting loss of molecules is thus expected to depend strongly on the longitudinal velocity of the molecules.

In Fig. 7, the maximum signal intensity of decelerated packets of $\mathrm{OH}$ radicals is shown at the exit of the 316 stage decelerator, operating in the $s=3$ mode. Beams of $\mathrm{OH}$ radicals with three different initial velocities have been used, and the signal is shown as a function of the final velocity (upper panel) or as a function of the phase angle (lower panel). When $\mathrm{Xe}$ or $\mathrm{Kr}$ are used as carrier gas, the Stark decelerator is programmed to select a packet of molecules with an initial velocity that is identical to the mean velocity of the molecular beam, i.e., 350 or $430 \mathrm{~m} / \mathrm{s}$, respectively. For Ar, a velocity of $520 \mathrm{~m} / \mathrm{s}$ is selected from the slow tail of the velocity distribution of the beam. The phase angle $\phi_{0}$ is varied to decelerate the selected packet of molecules to final velocities down to $100 \mathrm{~m} / \mathrm{s}$. The series of measurements for each seed gas are normalized to the data point that corresponds to $\phi_{0}$ $=0^{\circ}$. The thresholds are found at a velocity of about $150 \mathrm{~m} / \mathrm{s}$ in each series, and are indicated by the vertical dashed lines. This velocity is reached when $\phi_{0}=27^{\circ}, 43^{\circ}, 67^{\circ}$ for $\mathrm{Xe}, \mathrm{Kr}$, and Ar seeded beams, respectively. The value of the threshold velocity appears independent from the phase angle $\phi_{0}$ of the decelerator, consistent with the qualitative picture described above. The intensities that result from numerical trajectory simulations of the experiments are shown as solid lines in Fig. 7. Excellent agreement is obtained with the experiments and in particular the threshold behavior of the signal intensity is reproduced well.

The threshold velocity below which losses due to excessive transverse focusing occurs can be approximately determined as described in Appendix B. The model described there predicts that no stable trajectories exist in the $s=3$ mode of operation when $\lambda \leqslant(2.6 \times 2 s L)$. For the present Stark decelerator, operating on $s=3$, the periodicity of the transverse focusing force $2 s L$ is equal to $49.5 \mathrm{~mm}$. The mean transverse oscillation frequency $\Omega_{y}$ follows from the timeaveraged transverse force (see Appendix A). For $s=3, \Omega_{y}$ is rather independent from the phase angle $\phi_{0}$, and a threshold velocity of about $92 \mathrm{~m} / \mathrm{s}$ is found, consistent with the experimental findings. When the decelerator is operated in the $s=1$ mode, a threshold velocity is not so clearly defined, but similar losses due to excessive transverse focusing occur for velocities below $30 \mathrm{~m} / \mathrm{s}$.

The rather high threshold velocity for $s=3$ does not severely affect experiments in which Stark-decelerated beams are used for high-resolution spectroscopy and collision studies, or in which the decelerated beams are injected into molecular storage rings or synchrotrons. It does affect, however, experiments in which lower final velocities are required, e.g., trap loading experiments. There are several approaches to yet produce decelerated packets at a velocity below the threshold velocity with decelerators that are intended to operate at $s$ $=3$. An electric field geometry for the last section of the decelerator can be designed that permits a gradual reduction of the transverse focusing strength. This can be achieved by a dedicated electrode geometry and/or by a sequential reduction of the voltage that is applied to the electrodes. It is noted that similar strategies have already been implemented in trapping experiments using decelerators in the $s=1$ mode [20]. An alternative approach is to develop an electrode geometry for the last segment of the decelerator that allows the confinement of molecules in a genuine traveling potential well. When the velocity of this well is gradually reduced, the packet of molecules can be transferred from the threshold velocity to lower velocities without loss. The trapping of molecules in genuine traveling potential wells has already been demonstrated using optical fields [22] and using electric fields above a microstructured electrode array [23].

Within the possibilities of the present experimental arrangement, decelerated packets with low final velocities can be produced by changing over from the $s=3$ mode to the $s$ $=1$ mode before the threshold velocity has been reached. The number of molecules that exit the decelerator at velocities below the threshold velocity depends strongly on the details of the changeover, i.e., the velocity after which $s=1$ operation is used, and the phase angles that are used before and after the transition. The influence of the choice of these parameters on the number of molecules that exit the decelerator 
has been studied experimentally, decelerating a packet of $\mathrm{OH}$ radicals with an initial velocity of $350 \mathrm{~m} / \mathrm{s}$. The velocity and position of the packet in the decelerator at which the changeover from $s=3$ to 1 is made are systematically varied for the target (final) velocities of 100 and $50 \mathrm{~m} / \mathrm{s}$. For both velocities, the maximum signal intensity is observed when the transition to $s=1$ is made when the first $\sim 300$ stages are operated at $s=3, \phi_{0} \sim 26^{\circ}$ and when the molecular packet has reached a velocity of $170 \mathrm{~m} / \mathrm{s}$. The remaining 12 and 15 stages are then used at $s=1, \phi_{0}=43.2^{\circ}$ and $s=1, \phi_{0}=48.3^{\circ}$ to produce the final velocities of 100 and $50 \mathrm{~m} / \mathrm{s}$, respectively. The gain of this bimodal operation of the Stark decelerator with respect to $s=1$ operation is shown in Fig. 5 as separate data points. It is observed that for a final velocity of $100 \mathrm{~m} / \mathrm{s}$, the gain is about a factor of 2 , and is close to 1 for a final velocity of $50 \mathrm{~m} / \mathrm{s}$. These measurements demonstrate that low final velocities can be produced with Stark decelerators that are designed to operate at $s=3$, although the efficiency approaches the efficiency of conventional Stark decelerators when the target velocity is much lower than the $s=3$ threshold velocity.

\section{NUMERICAL TRAJECTORY CALCULATIONS}

The measurements presented thus far demonstrate the performance of the $s=1$ and 3 modes of operation of Stark decelerators in the (limited) range of parameters that is accessible to the experiment. In this section, both modes of operation are studied in a wider parameter range using numerical trajectory simulations. The electrode geometry that is used in the simulations is the same as used in the experiments, but the decelerator is allowed to have an arbitrary length.

Trajectories of $\mathrm{OH}$ radicals through the decelerator are numerically calculated as a function of the phase angle $\phi_{0}$ for both the $s=1$ and the $s=3$ mode of operation. In these simulations, a large number of molecules is homogeneously distributed at the entrance of the Stark decelerator over a block in 6D phase space. This block has a dimension of $20 \mathrm{~mm} \times 90 \mathrm{~m} / \mathrm{s}$ in the longitudinal direction, and a dimension of $4 \mathrm{~mm} \times 25 \mathrm{~m} / \mathrm{s}$ in each transverse direction. The molecular distribution has a mean forward velocity of $550 \mathrm{~m} / \mathrm{s}$ at the entrance of the decelerator, and is decelerated or accelerated to a final velocity of 180 or $755 \mathrm{~m} / \mathrm{s}$, corresponding to a change in the kinetic energy of $90 \%$. These values are arbitrary and can be chosen without loss of generality, as the phase-space acceptance of a Stark decelerator is in principle independent of the absolute initial and final velocity. The rather high final velocity of $180 \mathrm{~m} / \mathrm{s}$ for deceleration is chosen to stay away from the velocities for which excessive transverse focusing occurs, as discussed in Sec. II D. Decelerators containing 781, 388, 256, 190, 151, 126, 111, 102, and 99 electric field stages are simulated that are operated using $\left|\phi_{0}\right|=10^{\circ}, 20^{\circ}, 30^{\circ}, 40^{\circ}, 50^{\circ}, 60^{\circ}, 70^{\circ}, 80^{\circ}$, and $90^{\circ}$, respectively. These numbers apply to $s=1$; for simulations that apply to $s=3$, the number of stages is three times as large. For both values of $s$, additional simulations were performed for $\phi_{0}=0^{\circ}$ using a 2500 stages long decelerator. In each simulation, a sufficient number (5000 000 for $s=1$ and
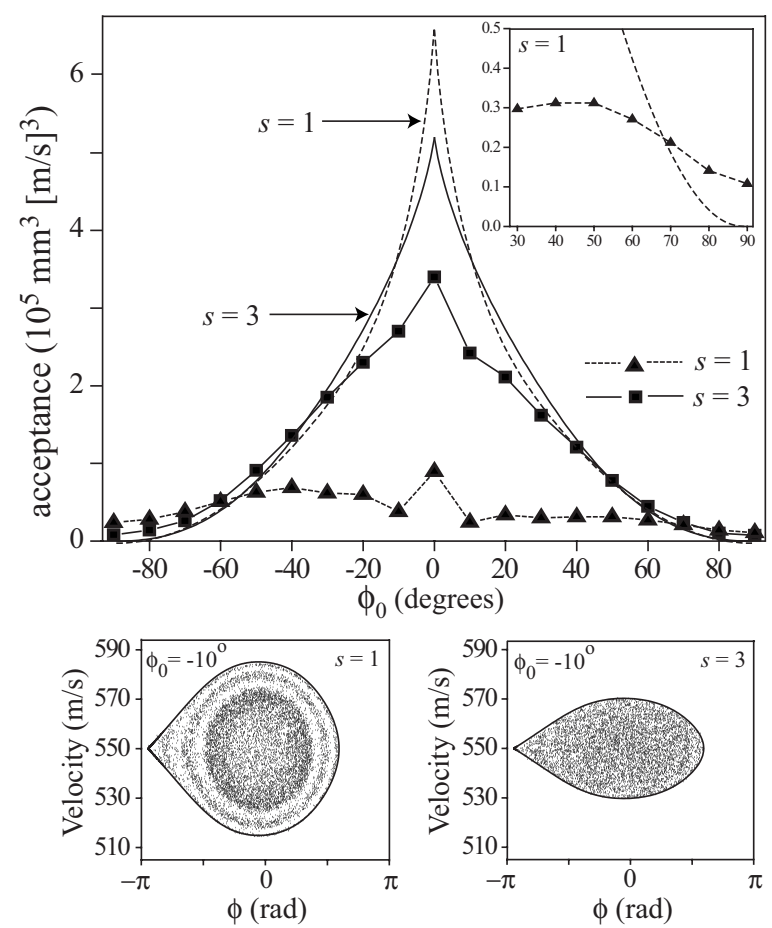

FIG. 8. 6D phase-space acceptance of a decelerator as a function of the phase angle $\phi_{0}$, resulting from numerical trajectory calculations (squares connected with solid lines for $s=3$; triangles connected with dashed lines for $s=1$ ), together with the model predictions (solid line for $s=3$; dashed line for $s=1$ ). In the inset, the $s$ $=1$ data are shown enlarged. In the lower part, the longitudinal phase-space distributions that result from the simulations are shown for $\phi_{0}=-10^{\circ}$, both for $s=1$ and 3 .

500000 for $s=3$ ) of molecules is generated to obtain good statistics. The number of molecules that are within the phasespace distributions of the decelerated packet are counted, and the corresponding 6D volume in phase space is calculated. In the lower part of Fig. 8, the simulated longitudinal phasespace distributions for $\phi_{0}=-10^{\circ}$ are shown both for $s=1$ and for $s=3$, together with the separatrices that follow from the 1D model for phase stability [19]. These distributions are representative for the distributions at low phase angles in general, and are shown here to exemplify the simulation method only. The phase-space distribution for $\phi_{0}=-10^{\circ}, \mathrm{s}$ $=1$ is highly structured with alternating stable and unstable regions. In the distribution for $\phi_{0}=-10^{\circ}, s=3$ no clear structure is present. The area within the longitudinal separatrixthe longitudinal acceptance-is a factor $\sqrt{3}$ smaller for $s=3$ than for $s=1$ [19].

In the upper panel of Fig. 8, the resulting simulated 6D phase-space acceptance is shown as a function of $\phi_{0}$ for both $s=1$ and 3. For $s=1$, the phase-space acceptance is maximum for $\phi_{0}=0^{\circ}$, and drops significantly for $\phi_{0} \neq 0^{\circ}$. It has a minimum at $\left|\phi_{0}\right|=10^{\circ}$, is rather constant for $20^{\circ} \leqslant\left|\phi_{0}\right|$ $\leqslant 50^{\circ}$, and drops again for $\left|\phi_{0}\right| \geqslant 50^{\circ}$, consistent with the experiments in Sec. II C. Note that the Stark decelerator has a nonzero acceptance for $\left|\phi_{0}\right|=90^{\circ}$, as discussed before [14]. It is interesting to note that the phase-space acceptance is not symmetric around $\phi_{0}=0^{\circ}$. The phase-space acceptance for $\phi_{0}<0$ is larger than the acceptance for the same positive 
phase angle; molecules spend less time in the decelerator when they are accelerated than when they are decelerated, reducing the loss due to instabilities.

The calculated acceptance for $s=3$ shows a rather different trend, and is generally larger for smaller values of $\left|\phi_{0}\right|$. Although the longitudinal phase-space acceptance for $s=3$ is a factor $\sqrt{3}$ smaller than for $s=1$, the 6D phase-space acceptance exceeds the acceptance for $s=1$ for $0^{\circ} \leqslant\left|\phi_{0}\right| \leqslant 70^{\circ}$. When $\left|\phi_{0}\right|=70^{\circ}$, the acceptance in both modes of operation is equal, consistent with the experimental findings discussed in Sec. II B. For $\left|\phi_{0}\right| \geqslant 70^{\circ}$, the acceptance for $s=1$ is slightly larger than the acceptance for $s=3$. Although less pronounced, the $\left|\phi_{0}\right|$ dependence of the acceptance for $s=3$ is again asymmetric around $\phi_{0}=0^{\circ}$.

It is interesting to compare the calculated phase-space acceptance with the phase-space acceptance that is expected for a Stark decelerator in which instabilities are absent. From this comparison, one can quantify the presence and severeness of instabilities in the $s=1$ and 3 mode of operation. The longitudinal phase-space acceptance of a decelerator is easily calculated and is, as mentioned above, simply given by the area within the separatrix $[11,19]$. The equations of motion that govern the transverse trajectories of molecules through the decelerator contain time-dependent forces, for which in general no simple analytical solutions exist. These equations, together with the equation for the longitudinal motion, can be used to estimate the volume in phase space from which stable trajectories can originate. This procedure is outlined in detail in Appendix A, and the resulting 6D phase-space acceptance is shown as a function of $\phi_{0}$ for both $s=1$ and 3 in Fig. 8. It is seen that for $s=1$, the phase-space acceptance predicted by the model deviates significantly from the calculated acceptance; for $\left|\phi_{0}\right| \leqslant 20^{\circ}$, the deviation is at least an order of magnitude. For larger angles the discrepancy gets less, and both curves cross around $\left|\phi_{0}\right|=70^{\circ}$. From this comparison, it is once more evident that instabilities are present when the decelerator operates in the $s=1$ mode [14], and that these instabilities severely limit the obtainable acceptance.

For the $s=3$ mode, the acceptance predicted by the model reproduces the calculated acceptance much better. The agreement for $\left|\phi_{0}\right|>40^{\circ}$ is good, and the deviations are in the $5-20 \%$ range for $20^{\circ} \leqslant\left|\phi_{0}\right| \leqslant 40^{\circ}$ and about $30 \%$ for $\left|\phi_{0}\right|$ $<20^{\circ}$. These minor deviations can be taken as an indication for the presence of small instable regions, as have indeed been observed close to the separatrix for $\phi_{0}=0^{\circ}$ [14]. The overall agreement, however, demonstrates that the 6D acceptance of a Stark decelerator in the $s=3$ mode of operation approaches the optimum value, i.e., the value that is predicted from the model that neglects any instabilities.

\section{CONCLUSIONS}

The studies presented in this paper address the question of how one can get the highest number density of decelerated molecules with a certain velocity at the exit of a decelerator. Rather than discussing a variety of electrode geometries that one might use to decelerate a beam of polar molecules, these studies focus on a Stark decelerator in the conventional, experimentally proven design. This decelerator can run at dif- ferent phase angles and operate in different modes, and can be built with a variable length. The number density of accelerated and decelerated $\mathrm{OH}$ radicals has been studied experimentally as a function of these three parameters. Quantitative comparisons of these number densities, obtained using Stark decelerators with different parameter sets, have been made. The measurements have been substantiated by numerical simulations, from which comparisons for a much wider range of parameters can be made. These studies provide quantitative arguments for the design criteria of Stark decelerators for specific applications.

Based on the one-dimensional description of a Stark decelerator, one would expect more molecules at the end of the decelerator for longer decelerators that run at lower phase angles. This description neglects the coupling between the longitudinal and transverse motion, however, which limits the actual 6D acceptance of a decelerator. A first important conclusion from the present study is that, for a decelerator operating in the $s=1$ mode, a strategy to optimize the number of decelerated molecules by using low phase angles and a large number of deceleration stages is only of limited use. There is a maximum of the 6D acceptance for a phase angle of around $50^{\circ}$, and the optimum number of molecules is obtained when the length of the decelerator (for a given initial and final velocity) is adjusted such that this phase angle can be used. A decelerator of 150 stages that is operated at $50^{\circ}$, for instance, produces more decelerated molecules than a decelerator of 250 stages that runs at $30^{\circ}$.

A second important conclusion is that a decelerator that operates in the $s=3$ mode outperforms a decelerator in the $s=1$ mode in almost all cases. In the $s=3$ mode, coupling between the longitudinal and transverse motion is nearly absent, and lower phase angles always result in a larger acceptance. For small phase angles, a gain up to a factor of 10 can be obtained. The gain depends strongly on the phase angle that is used, and for phase angles above $70^{\circ}$, the acceptance for the two modes of operation is very similar. An intrinsic disadvantage of the $s=3$ mode is that there are large losses for final velocities below around $150 \mathrm{~m} / \mathrm{s}$. Lower velocities can still be produced, however, and different schemes have been discussed and demonstrated for this.

A third important conclusion is that the acceptance of a Stark decelerator operating in the $s=3$ mode approaches the optimum value. This conclusion is based on a comparison between the outcome of numerical trajectory calculations and the $6 \mathrm{D}$ acceptance that is derived from a model. In this model, couplings between the longitudinal and transverse motion are neglected.

To make use of the advantages that the $s=3$ mode of operation offers, a considerably longer Stark decelerator is needed than for the $s=1$ mode. This indeed requires more electrode pairs and a longer vacuum chamber, but it should be realized that there is no additional requirement on the high voltage electronics. Compared to the decelerators that have been commonly used so far $\left(s=1, \approx 100\right.$ stages, $\phi_{0}$ $=50^{\circ}-60^{\circ}$ ), a five times longer version operating in the $s$ $=3$ mode at somewhat lower phase angles will typically result in a factor 5 higher number density at the exit. Moreover, this gain in number density is accompanied by a reduction in the longitudinal translational temperature. 


\section{ACKNOWLEDGMENTS}

This work is supported by the ESF EuroQUAM programme, and is part of the CoPoMol (Collisions of Cold Polar Molecules) project. The expert technical assistance of Georg Hammer, Manfred Erdmann, Rolf Meilicke, and the FHI mechanical and electronics workshops, as well as fruitful discussions with Hendrick L. Bethlem, are gratefully acknowledged.

\section{APPENDIX A}

In previous work, we have studied the transverse stability in a Stark decelerator [14]. Here we adapt the model for the transverse motion of molecules through a Stark decelerator, with the goal to derive the $6 \mathrm{D}$ phase-space acceptance as a function of the phase angle $\phi_{0}$ that can be expected when the presence of instabilities is neglected.

In the description of the motion of the $\mathrm{OH}$ radicals through the decelerator, the $z$ coordinate describes the position of the molecule along the molecular beam axis, while $x$ and $y$ are the transverse coordinates. The forces in the $x$ and the $y$ direction are assumed to be uncoupled from each other and identical. The alternating focusing in either one of the transverse directions (say $y$ ) is represented by a continuously acting transverse force $\bar{F}_{y}(\phi, y)$ that depends on the phase $\phi$ of the molecule and on $y[14]$,

$$
\begin{aligned}
\bar{F}_{y}(\phi, y) & =\frac{1}{2 T} \int_{t}^{t+2 T} F_{y}\left(y\left(t^{\prime}\right), z\left(t^{\prime}\right), t^{\prime}\right) d t^{\prime} \\
& \approx \frac{1}{2 s L} \int_{\phi L / \pi}^{(\phi+2 s \pi) L / \pi} F_{y}(y, z) d z,
\end{aligned}
$$

where $2 T$ is the time during which the synchronous molecule travels a distance $2 s L$.

To a good approximation, the transverse force $\bar{F}_{y}$ is linear in the displacement $y$ from the molecular beam axis. The strength of the transverse force can be expressed in terms of a frequency $\omega_{y}(\phi) / 2 \pi$, referred to hereafter as the natural transverse oscillation frequency, using the relation

$$
\bar{F}_{y}(\phi, y)=-m \omega_{y}^{2}(\phi) y,
$$

where $m$ is the mass of the $\mathrm{OH}$ radical. In Fig. 9, the natural transverse oscillation frequency is shown for an $\mathrm{OH}\left(X^{2} \Pi_{3 / 2}\right.$, $\left.J=3 / 2, M_{J} \Omega=-9 / 4\right)$ radical as a function of its phase $\phi$. For $s=1$, the natural transverse oscillation frequency has a strong dependence on the phase $\phi$. For molecules close to $\phi=0^{\circ}$, the transverse frequency is very low and focusing forces are almost absent. For $s=3$, the natural transverse oscillation frequency is rather independent from the phase $\phi$. The intermediate electric field stage focuses all molecules equally efficiently, effectively decoupling the transverse focusing properties of the decelerator from the longitudinal phase $\phi$.

The transverse phase-space acceptance for a given mode of operation of the decelerator is evaluated as schematically illustrated in Fig. 10. The decelerator is operated at $\phi_{0}$ $=30^{\circ}$ and $s=1$ in this example. Let us consider a molecule with a maximum deviation $z_{i}$ from the synchronous mol-

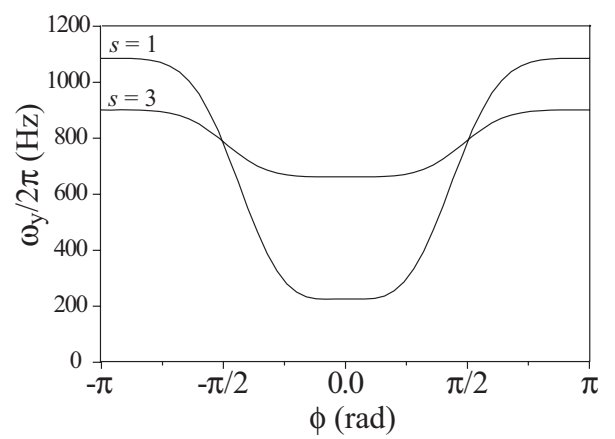

FIG. 9. Natural transverse oscillation frequency $\omega_{y} / 2 \pi$ for an $\mathrm{OH}\left(X^{2} \Pi_{3 / 2}, J=3 / 2, M_{J} \Omega=-9 / 4\right)$ radical as a function of its phase $\phi$ in a Stark decelerator, for the operation modes $s=1$ and 3 .

ecule. The frequency $\omega_{y}(t)$ of this molecule can be constructed from its phase $\phi(t)$ as it revolves around the synchronous molecule in longitudinal phase space. Let us now consider an ensemble of molecules that is enclosed by this contour, and by a contour that is displaced by an infinitesimal value $\Delta z_{i}$. All these molecules experience the same temporal dependence of the transverse focusing force, and the transverse trajectories of the molecules are governed by the equation

$$
\frac{d^{2} y}{d t^{2}}+\omega_{y}^{2}(t) y=0 .
$$

The transverse phase-space acceptance is easily calculated only if $\omega_{y}^{2}(t)$ is constant. In this case, in which $\omega_{y}(t)$ is written as $\omega_{y}$, the longitudinal and transverse motions are uncoupled, and in each transverse direction the molecules orbit ellipses in transverse phase space, as is shown in Fig. 10. The phase-space acceptance $\left(A_{y}\right)_{z_{i}}$ and $\left(A_{x}\right)_{z_{i}}$ in each transverse direction is given by the maximum extension $y_{\max }=x_{\max }$ $=1.5 \mathrm{~mm}$ from the molecular beam axis, and by the maximum transverse velocity $v_{y, \max }=v_{x, \max }=\omega_{y} \times y_{\max }$ that can be captured. The $4 \mathrm{D}$ volume $\left(A_{t}\right)_{z_{i}}$ of the transverse phase-space acceptance is then given by

$$
\left(A_{t}\right)_{z_{i}}=\left(A_{x}\right)_{z_{i}}\left(A_{y}\right)_{z_{i}}=\left[\pi \omega_{y}\left(y_{\max }\right)^{2}\right]^{2} .
$$

If $\omega_{y}^{2}(t)$ is not constant, as is actually the case in a Stark decelerator, the molecules experience a transverse frequency $\omega_{y}(t)$ that oscillates between the minimum value $\omega_{y}^{\min }$ and the maximum value $\omega_{y}^{\max }$. The time-averaged value of $\omega_{y}^{2}$ for this molecule is given by

$$
\left\langle\omega_{y}^{2}\right\rangle_{z_{i}}=\frac{1}{\tau} \oint \omega_{y}^{2}(t) d t,
$$

where $\tau$ is the time it takes the molecule to revolve the contour in longitudinal phase space. The transverse phase-space acceptance cannot be calculated anymore, but the values of $\omega_{y}^{\min }, \omega_{y}^{\max }$, and $\sqrt{\left\langle\omega_{y}^{2}\right\rangle_{z_{i}}}$ can nevertheless be used to characterize the transverse phase-space acceptance of the ensemble of molecules in three limiting cases. When the longitudinal oscillation frequency is much larger than the transverse oscillation frequency, $\omega_{y}$ can be taken as $\sqrt{\left\langle\omega_{y}^{2}\right\rangle_{z_{i}}}$. In that case, one obtains the 4D transverse acceptance for the molecules 


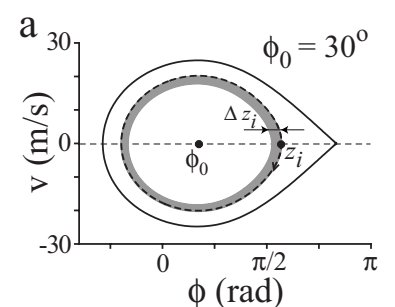

c

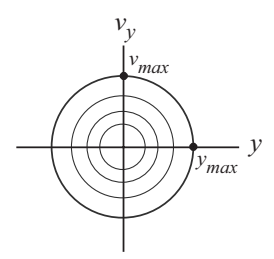

b

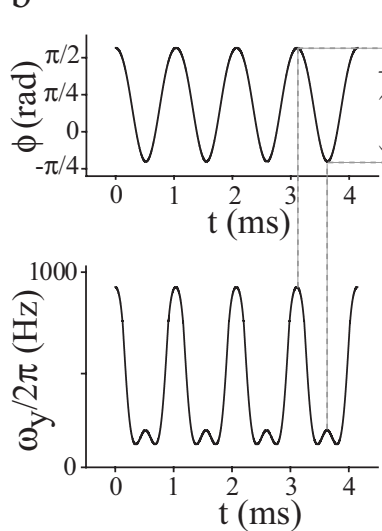

FIG. 10. Schematic representation of the method used to calculate the transverse phasespace acceptance. (a) The trajectory of a molecule in longitudinal phase space, shown as a dashed line. (b) The time dependence of the natural transverse oscillation frequency is constructed from the time dependence of the phase $\phi(t)$ and the phase dependence of the natural transverse oscillation frequency $\omega_{y}(\phi) / 2 \pi$. (c) The timeaveraged transverse focusing force results in elliptical orbits in transverse phase space.

in this shell in longitudinal phase space. This 4D acceptance can be interpreted as the best estimate for the true transverse acceptance. When $\omega_{y}^{\max }$ is used, a transverse acceptance results that can be interpreted as a strict upper limit for the true acceptance.

The total 6D phase-space acceptance $A\left(\phi_{0}\right)$ is obtained by integrating over all shells with area $d A_{z}$ within the separatrix in longitudinal phase space,

$$
A\left(\phi_{0}\right)=\int\left(A_{y}\right)_{z_{i}}\left(A_{x}\right)_{z_{i}} d A_{z} .
$$

In Fig. 11, the longitudinal (2D), transverse (2D), and total (6D) phase-space acceptances are shown for the operation modes $s=1$ and 3. The transverse acceptance $A_{y}\left(\phi_{0}\right)$ is calculated from the total 6D acceptance $A\left(\phi_{0}\right)$ and the longitudinal acceptance $A_{z}\left(\phi_{0}\right)$ via $A_{y}\left(\phi_{0}\right)=\sqrt{A\left(\phi_{0}\right) / A_{z}\left(\phi_{0}\right)}$. For each mode of operation, three curves are shown: the lower, center, and upper curve correspond to the choice of $\omega_{y}^{\min }$, $\sqrt{\left\langle\omega_{y}^{2}\right\rangle_{z}}$, and $\omega_{y}^{\max }$ for $\omega_{y}$ in Eq. (A4), respectively. The longitudinal acceptance $A_{z}\left(\phi_{0}\right)$ is a factor $\sqrt{3}$ smaller for $s=3$ than for $s=1$. The transverse acceptance for $s=3$ is somewhat larger than for $s=1$ and is almost independent of $\phi_{0}$. Furthermore, the three different curves predict a rather similar transverse acceptance for $s=3$. The three curves for the transverse acceptance for $s=1$ differ much more among each other. The center curves that are shown in Fig. 11(c), and that have been used in Fig. 8 of Sec. III, predict a rather similar total 6D phase-space acceptance for $s=1$ and 3. For almost all phase angles, the lower longitudinal phase-space acceptance for $s=3$ is compensated for by the larger transverse acceptance of $s=3$.

\section{APPENDIX B}

To obtain a more quantitative value for the threshold velocity below which excessive focusing occurs in the $s=3$ mode, we model the motion of a molecule with a longitudinal velocity that corresponds to the mean longitudinal velocity $\left\langle v_{z}\right\rangle$ of the ensemble. This molecule experiences a timeaveraged harmonic transverse force with a force constant $m\left\langle\omega_{y}^{2}\right\rangle$. This force constant is deduced from the transverse acceptance $A_{y}\left(\phi_{0}\right)$ [center curve for $s=3$ in Fig. 11(b) in Appendix A] via $m\left\langle\omega_{y}^{2}\right\rangle=m A_{y}^{2}\left(\phi_{0}\right) /\left(\pi y_{\text {max }}^{2}\right)^{2}$, and can be in- terpreted as the weighed average force constant of the ensemble of molecules in the packet.

The transverse force on a molecule in the decelerator has periodicity $2 s L$, and is modeled here by a square wave. In a given transverse direction, the molecule repeatedly experiences a constant transverse focusing force with harmonic fre-
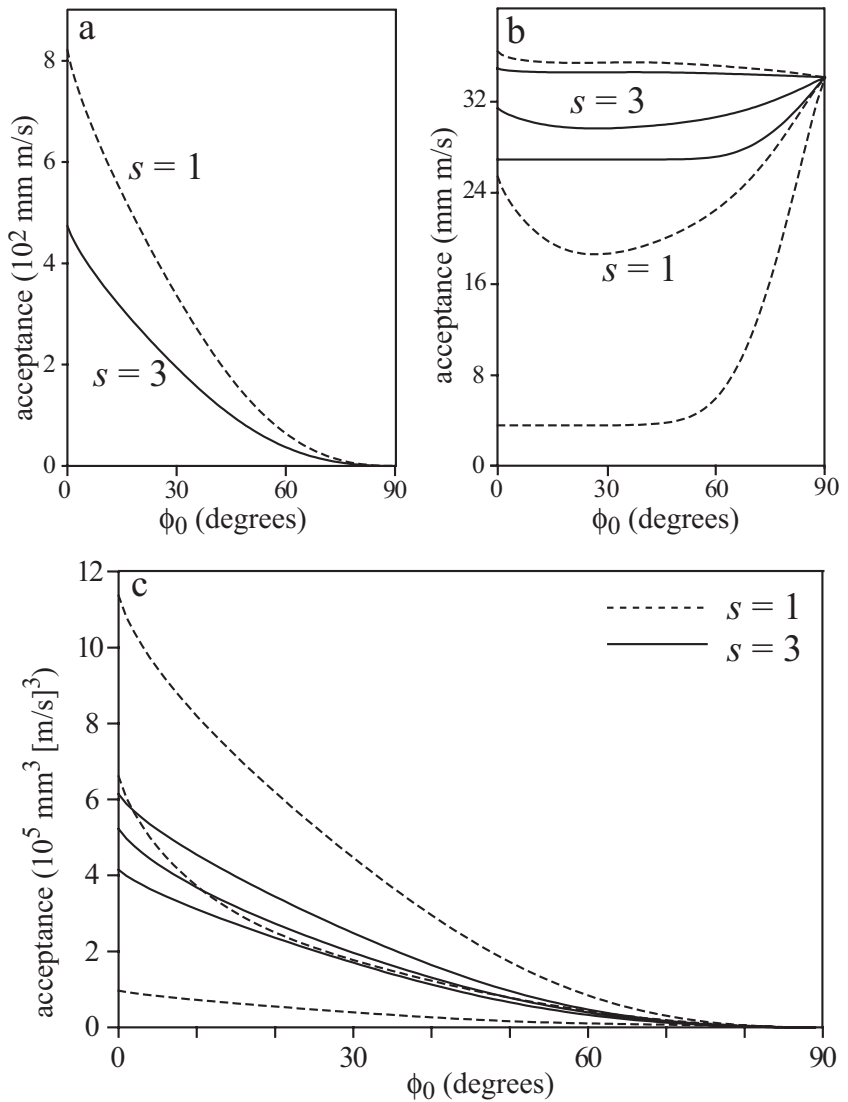

FIG. 11. Prediction for the longitudinal (a), transverse (b), and total 6D (c) phase-space acceptance of a Stark decelerator for the operation modes $s=1$ (dashed curves) and $s=3$ (solid curves) as a function of the phase angle $\phi_{0}$. For the transverse and 6D acceptances, three curves are shown for each mode of operation that predict the phase-space acceptance in three limiting cases, as explained in the text. The center curves describe the best estimate for the acceptance in the limit that instabilities in the decelerator can be neglected, and have been used in Fig. 8 of Sec. III. 
quency $\Omega_{y}$ followed by a section of free flight. The sections for focusing and free flight have equal length $s L$, such that $\Omega_{y}^{2}=2\left\langle\omega_{y}^{2}\right\rangle$.

For a constant velocity $\left\langle v_{z}\right\rangle$, the resulting equation of motion is of the type of Hill's differential equation, and the stability of transverse trajectories can be predicted with the transfer matrix method. The transformation matrices of the coordinate $y$ and velocity $v_{y}$ for the free flight and focusing sections are given by

$$
\left(\begin{array}{c}
y_{f} \\
v_{y_{f}}
\end{array}\right)=\left(\begin{array}{ll}
1 & T \\
0 & 1
\end{array}\right)\left(\begin{array}{c}
y_{i} \\
v_{y_{i}}
\end{array}\right)
$$

and

$$
\left(\begin{array}{c}
y_{f} \\
v_{y_{f}}
\end{array}\right)=\left(\begin{array}{cc}
\cos \left(\Omega_{y} T\right) & \frac{1}{\Omega_{y}} \sin \left(\Omega_{y} T\right) \\
-\Omega_{y} \sin \left(\Omega_{y} T\right) & \cos \left(\Omega_{y} T\right)
\end{array}\right)\left(\begin{array}{c}
y_{i} \\
v_{y_{i}}
\end{array}\right),
$$

respectively, where $T=s L /\left\langle v_{z}\right\rangle$ and where the subscripts $i$ and $f$ refer to initial and final coordinates, respectively. The transformation matrix $M$ for one full period is obtained by multiplication of the free flight and focusing matrices. The trajectories of the molecules through an infinite number of these periods are stable if $-2 \leqslant \operatorname{Tr}(M) \leqslant 2$, where $\operatorname{Tr}(M)$ is the trace of the transformation matrix [24]. When $\operatorname{Tr}(M)$ is outside this range, the amplitude of the transverse motion grows without bound.

In a Stark decelerator, the mean velocity $\left\langle v_{z}\right\rangle$ of the packet of molecules is not constant but is gradually changed. As long as the velocity change per electric field stage is modest compared to the longitudinal velocity, the assumptions in the model hold. For phase angles in the range of $30^{\circ}-40^{\circ}, \Omega_{y}$ is found as $\sqrt{2} \times 2 \pi \times 720 \mathrm{~Hz}$. For this value of $\Omega_{y}$, the inequality

$$
\left|2 \cos \left(\Omega_{y} T\right)-\Omega_{y} T \sin \left(\Omega_{y} T\right)\right| \leqslant 2
$$

implies that transverse trajectories are not stable for velocities $\left\langle v_{z}\right\rangle$ below $92 \mathrm{~m} / \mathrm{s}$. Or, in other words, the characteristic wavelength $\lambda$ for the transverse oscillation, given by $\lambda$ $=2 \pi\left\langle v_{z}\right\rangle / \Omega_{y}$, has to be larger than $2.6 \times 2 s L$ to obtain stable transverse trajectories.
[1] H. L. Bethlem, G. Berden, and G. Meijer, Phys. Rev. Lett. 83, 1558 (1999).

[2] S. Y. T. van de Meerakker, H. L. Bethlem, and G. Meijer, Nat. Phys. 4, 595 (2008).

[3] J. van Veldhoven, J. Küpper, H. L. Bethlem, B. Sartakov, A. J. A. van Roij, and G. Meijer, Eur. Phys. J. D 31, 337 (2004).

[4] E. R. Hudson, H. J. Lewandowski, B. C. Sawyer, and J. Ye, Phys. Rev. Lett. 96, 143004 (2006).

[5] J. J. Gilijamse, S. Hoekstra, S. Y. T. van de Meerakker, G. C. Groenenboom, and G. Meijer, Science 313, 1617 (2006).

[6] H. L. Bethlem, G. Berden, F. M. H. Crompvoets, R. T. Jongma, A. J. A. van Roij, and G. Meijer, Nature 406, 491 (2000).

[7] S. Y. T. van de Meerakker, P. H. M. Smeets, N. Vanhaecke, R. T. Jongma, and G. Meijer, Phys. Rev. Lett. 94, 023004 (2005).

[8] S. Y. T. van de Meerakker, N. Vanhaecke, M. P. J. van der Loo, G. C. Groenenboom, and G. Meijer, Phys. Rev. Lett. 95, 013003 (2005).

[9] S. Hoekstra, J. J. Gilijamse, B. Sartakov, N. Vanhaecke, L. Scharfenberg, S. Y. T. van de Meerakker, and G. Meijer, Phys. Rev. Lett. 98, 133001 (2007).

[10] J. J. Gilijamse, S. Hoekstra, S. A. Meek, M. Metsälä, S. Y. T. van de Meerakker, G. Meijer, and G. C. Groenenboom, J. Chem. Phys. 127, 221102 (2007).

[11] H. L. Bethlem, F. M. H. Crompvoets, R. T. Jongma, S. Y. T. van de Meerakker, and G. Meijer, Phys. Rev. A 65, 053416
(2002).

[12] M. Baranov, L. Dobrek, K. Góral, L. Santos, and M. Lewenstein, Phys. Scr., T T102, 74 (2002).

[13] H. L. Bethlem, G. Berden, A. J. A. van Roij, F. M. H. Crompvoets, and G. Meijer, Phys. Rev. Lett. 84, 5744 (2000).

[14] S. Y. T. van de Meerakker, N. Vanhaecke, H. L. Bethlem, and G. Meijer, Phys. Rev. A 73, 023401 (2006).

[15] J. G. Kalnins, G. Lambertson, and H. Gould, Rev. Sci. Instrum. 73, 2557 (2002).

[16] B. C. Sawyer, B. K. Stuhl, B. L. Lev, J. Ye, and E. R. Hudson, Eur. Phys. J. D 48, 197 (2008).

[17] S. Y. Lee, Accelerator Physics (World Scientific, Singapore, 1999).

[18] R. Wolfgang, Sci. Am. 219 (4), 44 (1968).

[19] S. Y. T. van de Meerakker, N. Vanhaecke, H. L. Bethlem, and G. Meijer, Phys. Rev. A 71, 053409 (2005).

[20] S. Y. T. van de Meerakker, N. Vanhaecke, and G. Meijer, Annu. Rev. Phys. Chem. 57, 159 (2006).

[21] J. Küpper, H. Haak, K. Wohlfart, and G. Meijer, Rev. Sci. Instrum. 77, 016106 (2006).

[22] R. Fulton, A. I. Bishop, M. N. Shneider, and P. F. Barker, Nat. Phys. 2, 465 (2006).

[23] S. A. Meek, H. L. Bethlem, H. Conrad, and G. Meijer, Phys. Rev. Lett. 100, 153003 (2008).

[24] E. D. Courant and H. S. Snyder, Phys. Rev. 88, 1190 (1952). 